\title{
The 49th Annual Meeting of the German Society of Neuroradiology, Cologne, October 23rd to 25th, 2014
}

\section{U. Ernemann}

Published online: 24 May 2014

(C) Springer-Verlag Berlin Heidelberg 2014

Dear colleagues and friends of neuroradiology,

It is a great pleasure to invite you to the annual meeting of the German Society of Neuroradiology, which will be held again in the familiar and welcoming setting of the Gürzenich Congress Centre in Cologne. The successful combination of neuroradiological state of the art teaching sessions, given by particularly renowned experts in their fields, with scientific sessions dedicated to the latest research by young and established scientists has turned this meeting into the most popular one in neuroradiology.

This year we are proud to announce for our meeting a scientific partnership with the German Cancer Society and the German Society of Skull Base Surgery which emphasizes the outstanding role of neuroradiology as a diagnostic and therapeutic partner in numerous clinical cooperations. These aspects will be addressed by teaching sessions which give an update on neuro-oncological imaging and skull base pathologies.

The rapidly evolving field of imaging technologies will be covered with a focus on new magnetic resonance imag- ing (MRI) methodology including the intraoperative setting as well as vascular and preclinical imaging.

Our interventional work will be reviewed with regard to current trials on intra-arterial stroke therapy and to the ongoing debate about treatment options for arterio-venous malformations.

With a teaching session on degenerative spinal disease and differential diagnoses we address an important segment of the clinical workload of our colleagues both in radiology and neuroradiology.

The dedicated program for radiology technicians is an outstanding landmark of neuroRAD 2014 and will cover clinically relevant aspects of oncological imaging, evolving MRI techniques, and pediatric neuroradiology.

Abstract submission is already open. For further information on neuroRAD 2014 please visit our website www. neurorad.de/.

The German Society of Neuroradiology would like to welcome you as a participant at its upcoming meeting from October 23rd to 25th, 2014 in Cologne.
U. Ernemann $(\bowtie)$

Department of Diagnostic and Interventional Neuroradiology,

University of Tübingen,

Tübingen, Germany

e-mail: Ulrike.Ernemann@med.uni-tuebingen.de 